\section{Intensive care}

\section{G76 INCIDENCE AND OUTCOME OF POST-MENINGITIC HYDROCEPHALUS}

K. Vijayakumar', J.R. Edwards, P. Prabhakar', I.K. Pople', P.J. Murphy², P.M. Sharples'. 'Department of Paediatric Neurology, Frenchay Hospital, Bristol; ${ }^{2}$ Paediatric Intensive Care Unit, Bristol Royal Hospital for Sick Children, UK

Introduction: Historically 1.9-2.8\% of children developed hydrocephalus following bacterial meningitis. The changes in incidence and outcome of post-meningitic hydrocephalus with modern pediatric intensive care unit (PICU) management of meningitis have not been clearly defined.

Methods: A regional PICU database, incorporating clinical, radiological and outcome (Glasgow outcome score (GOS)) data of all admissions (1997-2001) for severe bacterial meningitis ( $n=129)$ was interrogated and cross-referenced with all records of children undergoing treatment of post-meningitic hydrocephalus $(n=11)$ in the regional pediatric neurosurgical unit from 1997-2001. Regional figures for all cases of bacterial meningitis in children $(n=513)$ were obtained from the Communicable Disease Surveillance Center.

Results: Hydrocephalus requiring shunting complicated $2.1 \%$ of all cases of bacterial meningitis. Both early $(p=0.018)$ and late (shunted) $(p<0.001)$ hydrocephalus was significantly associated with young age (< 1 yr). In infants, hydrocephalus complicated significantly more cases of Group B Streptococcal $(5 / 18 ; 29 \%)$ and pneumococcal $(3 / 40 ; 7.5 \%)$ meningitis compared to meningococcal infections $(2 / 119 ; 1.7 \%)(p=0.004)$. Overall $16 \%(13 / 81)$ of all imaged PICU meningitis patients had radiological evidence of early hydrocephalus of whom $4(31 \%)$ required shunting; 2 of these had a poor neurological outcome (GOS 1-3). Neurological outcome in the entire shunted hydrocephalus population was poor (GOS $1-3$ ) in $6 / 11$ cases $(55 \%)$.

Conclusions: Post-meningitic hydrocephalus occurs almost exclusively in the infant population, reflecting the poor compensatory reserve due to the underdevelopment of the CSF pathways in this age group. Despite modern PICU management the overall incidence of post-meningitic hydrocephalus remains unchanged. The development of hydrocephalus is associated with a poor neurological outcome.

\section{G77 NEUROLOGICAL OUTCOME IN CHILDREN WITH NON-TRAUMATIC COMA ADMITTED TO A REGIONAL PAEDIATRIC INTENSIVE CARE UNIT}

K. Vijayakumar ${ }^{1}$, R. Knight ${ }^{1}$, P. Prabhakar' ${ }^{1}$ P.J. Murphy², P.M. Sharples' 'Department of Paediatric Neurology, Frenchay Hospital, Bristol; ${ }^{2}$ Department of Paediatric Intensive Care, Bristol Royal Hospital for Children

Introduction: Non-traumatic coma (NTC) is an important cause of mortality and morbidity in children. Centralisation of PIC services might be anticipated to result in improved outcome, but this issue has not hitherto been investigated.

Aims: To define the neurological outcome of all cases of NTC aged $\leqslant 16$ yrs admitted to a Regional PICU over the 5 year period 19972001 and to identify factors associated with poor outcome.

Method: Retrospective case notes review. Coma was defined as Glasgow Coma Score (GCS) <13 for more than 6 hours. Neurological outcome was defined using an adaptation of the Seisha Outcome Scale. Population data for the South West region was obtained from Office of National Statistics (ONS).

Results: 328 children were referred to the Regional PICU for NTC over the study period, an annual incidence of 7.05/100 000 children. 188 (57\%) were boys, 140 (43\%) girls. The median age was 2 yrs (range $0.01-16) .238$ children (72\%) were transferred from district hospitals. The median length of PICU stay was 3 days (range 1-64). 279 (85\%) required IPPV and 139 (42\%) inotropic support. $164(50 \%)$ had CNS infection, 88 (27\%) status epilepticus, 39 (12\%) toxic or metabolic encephalopathy, $13(4 \%)$ hypoxic-ischaemic encephalopathy, $21(6 \%)$ other encephalopathies and in $3(1 \%)$ the cause of the NTC was not identified. 206 children $(62 \%)$ had evidence of seizures; these were prolonged (>30 mins) in 120. Only 18 (5.4\%) underwent intracranial pressure (ICP) monitoring, but 234 $(72 \%)$ had clinical or other evidence to suggest raised ICP. 178 children $(54 \%)$ survived with good neurological outcome, $25(8 \%)$ with moderate or mild disability, $32(10 \%)$ with severe disability and 39 $(12 \%)$ died. Poor outcome was significantly related to young age $1<2$ yrs) ( $<<0.001)$, low GCS ( $\leqslant 8)(p<0.001)$, aetiology $(p<0.001)$, evidence suggesting raised ICP $(p<0.001)$ and inotropic requirement $(p<0.001)$. Multiple regression analysis showed evidence suggesting raised ICP and inotropic requirement to be independent predictors of poor outcome.

Conclusion: Our incidence of poor outcome (22\%) was considerably lower than that reported for 1994-1995 from the North East $(36 \%)$. We hypothesise that part of this difference might be due to centralisation and improved delivery of PIC.

\section{G78 DIAPHRAGMATIC PARALYSIS IN CHILDREN AFTER CARDIAC SURGERY}

V.P. Balasubramaniam, M. Burmester. Paediatric Intensive Care Unit, Royal Brompton Hospital, Sydney Street, London SW3 6NP

Aims: To determine the prevalence and clinical impact of diaphragmatic paralysis caused by phrenic nerve injury after cardiothoracic surgery in children.

Methods: A search of surgical and paediatric intensive care unit databases identified 12 episodes of diaphragmatic paralysis after cardiothoracic surgery in 398 children over a one-year period from 2001 to 2002. Medical records were reviewed to determine demographics, details of the operation and postoperative course, diagnostic features and management of diaphragmatic paralysis, and follow up status.

Results: The incidence of clinically apparent diaphragmatic paralysis was $3 \%$. The incidence following open procedures was $10 / 274(4(33 \%)$ of which had undergone the Switch procedure), following closed procedures 2/124. All injuries were unilateral with an equal incidence for each side. Median age at operation was 40 days (range 1 day-10 months). Diagnosis was made by ultrasound scan in all children, with diaphragmatic fluoroscopy confirming the ultrasonographic findings in one child. Median time from operation to investigation was 10 days (range 2-16 days) with 10 (83\%) children receiving mechanical ventilation at diagnosis, $50 \%$ of whom had required reintubation after extubation failure. All 10 of these children underwent diaphragmatic plication. Associated postoperative lesions included one case of vocal cord palsy and 2 cases of chylothorax. Median duration of invasive ventilation after cardiothoracic surgery was 8.5 days (range 2-60 days). Overall duration in intensive care was median of 28 days (range 4-90 days). Independent factors associated with increased postoperative hospital stay were lower patient weight at operation, previous cardiothoracic operations, mechanical ventilation at time of investigation, and diaphragmatic palsy.

Conclusion: Retrospective review for diaphragmatic paralysis revealed an incidence in children of less than 1 year of $3 \%$. Younger children requiring mechanical ventilation may present a higher risk group to target for increased diagnostic suspicion and more aggressive management. Active diagnostic exclusion of diaphragmatic palsy is recommended in cases of extubation failure.

\section{G79 PAEDIATRIC EARLY WARNING SYSTEM (PEW)-A MODEL FOR IMPROVED RECOGNITION AND MANAGEMENT OF THE CRITICALLY ILL}

S. Ferguson', M. Stark' , J. Madar' ${ }^{2}$ Departments of 'Intensive Care and ${ }^{2}$ Paediatrics, Derriford Hospital, Plymouth

The recognition and management of the critically ill child outside the intensive care setting is of critical importance. Delay leads to potentially avoidable morbidity and mortality. This abstract describes the development of a paediatric early warning system (PEW) within our hospital.

Method: PEW comprises a group of key physiological observations. These are classified along the A, B, C, and D approach. In addition a number of clinical conditions activate the system irrespective of physiological state. Activation leads to senior paediatric/intensive care review and the initiation of appropriate treatment.

A prospective evaluation of PEW through case note review for all consecutive admissions occurred over a 4-month period. Children attaining one or more PEW criteria were selected to determine if critically ill children were being correctly identified with subsequent activation of the PEW system, and if this led to a change in clinical management

Results: All 808 admissions in the time period were reviewed. 50 $(6.1 \%)$ attained 1 or more PEW criteria. PEW was activated in 35 $(70 \%)$. The mean time from presentation to PEW activation was 2.2 hours (0-23.25 hours). Commonest site of activation was Children's High Dependency 23/35 (65.7\%). Median time from PEW activation to senior clinician revue was 0.9 hours $(0.1-7$ hours). 
In $20(57.1 \%)$ of the activated cases, management changed following senior review. The remaining $15(42.8 \%)$ where merely highlighted as a potential problem with no intervention.

Conclusion: PEW is a model for the improved recognition and early management of the sick child. Although only a small proportion of paediatric admissions led to its activation, in more than half, early senior clinician involvement led to a change in clinical management and may have prevented morbidity or mortality

\section{G80 LONGTERM PSYCHOLOGICAL OUTCOME OF CHILDREN ADMITTED TO HOSPITAL WITH MENINGOCOCCAL DISEASE}

D. Shears, M.E. Garralda, S. Nadel, J. Gledhill. St. Mary's Hospital, London

Aims: To describe psychiatric adjustment 9-12 months after discharge in children admitted to hospital with meningococcal disease.

Methods: This cohort study includes children aged 3 to 16 admitted to 3 PICUs and 19 general paediatric wards in the London area. During the index admission, severity of illness is assessed with the The Glasgow Meningococcal Septicaemia Prognostic Score (GMSPS). Parents are interviewed and demographic details are obtained. The child's previous psychiatric adjustment is determined by parent and teacher completed Strengths and Difficulties (SDQ) questionnaires.

At 9-12 months follow-up childrens' psychiatric adjustment is assessed using a semi-structured diagnostic interview, the KiddieSADS (in children over 6 years) and The Behavioural Screening Questionnaire - BSQ, (in children under 6). Questionnaires are also completed by parents outlining the child's psychiatric adjustment since discharge using the SDQ; the presence of stress disorders is ascertained using the Impact of Event Scale (IES); the parents' psychiatric adjustment is measured with the General Health Questionnaire (GHQ). Symptoms of Post-traumatic Stress are assessed with the Impact of Event Scale.

Results: 67 out of 78 children and their parents were assessed at admission and after 9 months. Of the 67 children, 50 Children required PICU admission and 17 children were treated on a general paediatric ward. Mean age (s.d.) for the whole group was 7.97 (4.32). There were equal numbers of boys and girls. At 9 months there were significant rises in total deviance scores $(p=0.05)$ and emotional problems $(p=0.000)$ as measured by the SDQ. On the K-SADS $48.6 \%$ of children, over 6 , had developed psychiatric disorder at some point during the year following their admission. The main diagnoses, shortly after admission, were depressive disorders and oppositional defiant disorders. $24 \%$ had psychiatric disorder at 9-12 months, chiefly oppositional defiant disorder and anxiety disorders.

Conclusions: Admission to hospital with meningococcal disease may be associated with high rates of psychiatric disorder. Whilst depressive disorders predominate shortly after admission, oppositional disorders and anxiety disorders are the largest diagnostic group at a year.

\section{G81 ENTERAL FEEDING IN SICK CHILDREN: A BLIND TECHNIQUE FOR PLACING NASOJEJUNAL FEEDING TUBES}

R. Meyer, S. Harrison, K. Elwig, R. Nicholson, P. Ramnarayan. Dept. of Paediatrics, Dietetics, Clinical Physics at St Mary's Hospital, London W2 INY

Introduction: In critically ill children $(\mathrm{ClC})$, gastroparesis is a common complication preventing successful nasogastric feeding. Postpyloric feeding has been shown to be safe and effective in this subset of patients; however, placement has often been avoided or delayed due to poor migration of weighted tubes into the jejunum and the need for fluoroscopy and endoscopy procedures. These are expensive and involve significant risk to the patient.

Aims: To develop a reliable blind technique that can be used at the bedside and does not require exposure to radiographs for confirmation.

Methods: The tube selected for the procedure was a non-weighted polyurethane nasogastric tube with a flexible stylet. Tube placement took place whilst the child was in the right lateral oblique position. Confirmation of post pyloric placement relied on a combination of the following: length of tube, auscultation, $\mathrm{pH}$ of aspirate and a blue dye test. This technique was piloted on 50 patients admitted to a Paediatric Intensive Care Unit (PICU) over a period of ten months in 2000. Concurrent radiographs were obtained for confirmation of NJT position.
Results: The success rate of the initial pilot was $96 \%$. No complications related to placement were encountered. The blind bedside technique was then introduced for clinical use and supported by an intensive staff training programme. The technique was audited in 2001 indicating a $95.2 \%$ successful NJT placement rate. The mean time for successful placement was $28.8 \mathrm{~min}$ (range 5-75 min). None of the patients in 2001 required radiological confirmation of NJT position. The mean effective dose of radiation saved per abdominal radiograph for a 1 -year-old is $0.06 \mathrm{mSv}$ ( 9 days natural background radiation in the UK) and for a 5-year-old $0.12 \mathrm{mSv}$ (17 days natural background radiation in the UK).

Conclusions: In $\mathrm{ClC}$ who do not tolerate nasogastric feeding, enteral alimentation can be safely and successfully achieved by NJT placement at the bedside without the use of radiological confirmation.

\section{G82 LACTOBACILLUS CASEI SHIROTA (LCS) AS A PROBIOTIC IN CRITICALLY ILL CHILDREN (CIC)—IS IT SAFE?}

R. Srinivasan, R. Meyer, P. Ramnarayan, J. Britto. Dept. Paediatrics and Dietetics, Imperial College of Medicine at St Mary's Hospital, London W2 INY

Background: $\mathrm{CIC}$ with multisystem failure on PICU have been shown to benefit from early enteral feeding. Diarrhoea is frequently observed in this subset of patients. There is increasing evidence that probiotics decrease the incidence of nosocomial diarrhoea and in addition positively modulates host immunity. There are no reports on the use of probiotics - LCS in $\mathrm{ClC}$ and the safety of its use.

Aim: Establish safety (bacteriological cultures/colonisation) LCS on PICU.

Methods: Local ethics approval was awarded for the study. Prospective, descriptive pilot study on PICU. Children without documented immodeficiency and over 6 months of age were randomised to 2 groups. Study group: 3 doses of LCS (107 live culture) enterally per day for a maximum of five days Control group: standard enteral feed as per PICU protocol. Bacteriology provided support in the identification of cultures and colonisation.

Results: A total of 56 patients were recruited, 28 in each arm of the trial. Bacteriological studies on: secretion, blood cultures (peripheral/ vascular access) and urine were performed as per PICU protocol.

Mean ages of the study and control groups were 2.74 and 3.9 years respectively. The sex ratio (M:F) was 16:12, and 15:13. In both groups $9 / 28$ of children had a primary diagnosis of meningococcal disease. The most frequent organism obtained on blood culture was coagulase negative Staphylococcus, which was cultured from blood in 8 and 7 in the study and control groups. Lactobacillus was not detected on any of the bacgteriological specimens in either group. There were no clinically apparent side effects of LCS.

Conclusions: Our pilot study suggests that use of LCS as a probiotic- in enterally fed children with multisystem failure is safe. Further studies are currently under way to evaluate the impact of LCS on frequency and consistency of stools in $\mathrm{CIC}$.

\section{G83 IDENTIFICATION OF A NEW ROLE FOR IL-6 AS A MYOCARDIAL DEPRESSANT FACTOR IN MENINGOCOCCAL SEPTICAEMIA BY GENE EXPRESSION PROFILING USING CDNA MICROARRAYS}

N. Pathan, C. Hemingway, A.C. Stephens, E. Oragui, C. Sandiford, P. O'Gara, C. McCabe, S. Popper, D. Relman, S. Harding, M. Levin. Depts of Paediatrics and Cardiac Medicine, Imperial College and Infectious Diseases, Stanford University

Aims: Death from meningococcal disease is most often due to profound cardiovascular deterioration. Elucidation of the mechanisms leading to myocardial dysfunction in meningococcal shock could target novel therapies for this and other forms of septicaemia. We recently established that a 10-25 KDa heat-stable protein with myocardial depressant activity is released in meningococcal septicaemia.' We examined the entire inflammatory repertoire in meningococcal-activated whole blood using cDNA microarrays, to identify proteins that might be responsible for the myocardial depression.

Methods: Whole blood obtained from healthy volunteers was inoculated with live meningococci. RNA was isolated, amplified and hybridised to a customised DNA microarray. Genes that were significantly upregulated were scanned for those releasing proteins of 10-25 KDA. These proteins were further analysed for myocardial depressant activity using isolated cardiac myocytes as described.' 
Results: Of 141 genes that had $>1.5$ log increased in expression after 3 hours meningococcal whole blood stimulation, only 5 generated 10-25 KDa proteins: IL-1 $\beta$, TNF $\alpha$, IFN $\gamma$, IL-1 $\alpha$, and IL-6. We previously excluded myocardial depressant effects of the first 3 in meningococcal sepsis.' IL-1 $\alpha$ also had no depressant activity. IL-6 caused acute depression of isolated cardiac myocyte contractility in vitro (mean $-17.1 \%, p=0.002$ ). Removal of IL-6 from meningococcal serum completely reversed its negative inotropic activity. IL-6 levels in 38 patients were significantly associated with PRISM score $(p=0.0004)$ and inotrope requirement $(p<0.0001)$.

Conclusions: The myocardial dysfunction in meningococcal septicaemia is directly mediated by Interleukin 6 (IL-6). Using a combination of conventional protein purification and microarray technology, this study highlights a critical involvement of IL-6 in the pathogenesis of sepsis-associated cardiomyopathy, and implicates IL-6 as a potential target in the treatment of septic shock.

1. Pathan, N. et al. Crit Care Med 2002;2191-8.

\section{G84 THE EFFECT OF TEMPERATURE AND STRESS ON MYOCARDIAL TROPONIN I RELEASE IN NEWBORN PIGS EXPOSED TO ACUTE HYPOXIA ISCHAEMIA}

S. Satas', J. Tooley', M.S. Suleiman'2, M. Thoresen'. 'Dept of Child Health, St Michael's Hospital, University of Bristol; ${ }^{2}$ Bristol Heart Institute, Bristol Royal Infirmary

Background: Global hypoxia ischaemia $(\mathrm{HI})$ and reoxygenation in newborn pigs leads to brain damage and to disruption of normal function of other organs including the heart. There is evidence that local myocardial or whole body hypothermia provides myocardial protection during or after the onset of hypoxia/ischaemia. Myocardial Troponin I (Tnl) is a highly specific marker of myocardial damage.

Aim: To compare the extent of cardiac injury measured by release of Tnl associated with: a) normothermic (NT) or hypothermic (HT) reoxygenation with or without anaesthesia after acute $\mathrm{Hl}$; b) rewarming after HT.

Methods: Two series of experiments were performed exposing newborn pigs (age $7-48 \mathrm{~h}$ ) to $45 \mathrm{~min}$ of global $\mathrm{HI}$ followed by $24 \mathrm{~h}$ of NT (rectal temperature $39.0^{\circ} \mathrm{C}$ ) or mild HT $\left(34.5^{\circ} \mathrm{C}\right)$. Anaesthesia was used during recovery in 18 animals (total 54 pigs) but was stopped $24 \mathrm{~h}$ after the insult. A rewarming period of six hours followed in all animals. HR and MABP were monitored and recorded. Blood samples for $\mathrm{Tnl}$ were collected before $\mathrm{HI}$ and $6,12,24,30,48 \mathrm{~h}$ after $\mathrm{HI}$. Tnl was measured using ACCESS system (Beckman, Inc). The stress level in pigs was evaluated by measuring plasma cortisol.

Results: Global HI resulted in a significant $\mathrm{Tn}$ l release. In anaesthetised pigs Tnl increase was much higher in the NT than in the HT group

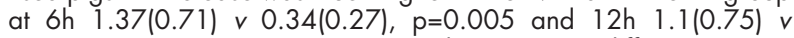
$0.37(0.23), p=0.027$, (mean (SD)). There was no difference in Tnl release between the non-anaesthetised NT and HT pigs Average HR during recovery was higher in anaesthetised NT $V$ HT animals, $199(15) \vee 143(21), p=0.0005$. Only in HT non-anaesthetised pigs did cortisol concentrations increase from 324(122) before $\mathrm{HI}$ to $751(271)$ at $24 \mathrm{~h}(\mathrm{p}=0.026)$. During $6 \mathrm{~h}$ rewarming Tnl rose in the HT pigs only in both series of experiments.

Conclusions: Global HI followed by reoxygenation led to a significant rise in serum $\mathrm{Tnl}$ in the newborn pig. Anaesthesia abolishes stress and release of $\mathrm{Tnl}$ observed in the HT pigs. Rewarming induced another release of $\mathrm{Tnl}$ suggesting additional myocardial damage.

\section{G85 THE STRESS RESPONSE AND LYMPHOCYTE APOPTOSIS IN ACUTE RESPIRATORY SYNCYTIAL VIRUS BRONCHIOLITIS}

M.F.E. Roe', R. Patel', D.K. White', D.M. Bloxham², R.I. Ross-Russell', R.C. Tasker', D.R. O'Donnell'. 'Department of Paediatrics and '2Department of Haematology, University of Cambridge

Respiratory syncytial virus (RSV) bronchiolitis is associated with an apoptosis-related lymphopaenia, most pronounced in the sickest children. We hypothesised that a neuroendocrine stress response may alter circulating lymphocyte susceptibility to apoptosis.
Aim: To examine the relationship between neuroendocrine stress hormones and apoptosis in RSV infection.

Methods: Blood was taken from 32 infants with RSV bronchiolitis and after recovery. Prolactin, cortisol, vasopressin and leptin levels were measured by ELISA. Plasma Fas ligand (FasL) was used as a surrogate for lymphocyte apoptosis.

Results: FasL levels were raised during acute bronchiolitis $(p=0.028)$. Cortisol $(p<0.001)$ and prolactin $(p=0.005)$, which may induce apoptosis, were increased during illness compared with recovery. Vasopressin levels were similar at both time points $(p=n s)$. Leptin, which may reduce apoptosis was reduced in acute illness $(p=0.007)$ especially in PICU patients ( $p=0.028$ )

Conclusions: These data suggest that in RSV infection, lymphopaenia of circulating lymphocytes may be altered by the neuroendocrine stress response.

Funding: 2002 HC Roscoe Fellowship, BMA.

\section{G86 NON-INVASIVE DIAGNOSIS OF} TRACHEOBRONCHOMALACIA IN PAEDIATRIC INTENSIVE CARE- A MODIFIED VENTILATION RADIOISOTOPE LUNG SCAN

A. Gour, I. Gordon, A.J. Petros. Paediatric Intensive Care Unit, Great Ormond Street Hospital for Children NHS Trust, London WCIN 3JH

Aim: To evaluate the usefulness of radio nucleotide ventilation scans in children with tracheobronchomalacia (TBM) on a Paediatric Intensive Care Unit (PICU)

Introduction: TBM is a significant cause of morbidity and prolonged stay on PICU. TBM may be primary or secondary to congenital heart disease, prolonged intubation, gastro oesophageal reflux or following surgery for repair of tracheo-oesophageal fistula. Tracheobronchogram (TBG) and bronchoscopy are established methods for diagnosing TBM.

Methods: Four intubated infants aged $<6$ months, with clinical TBM underwent TBG and isotope ventilation lung scans at incremental levels of peak end expiratory pressure (PEEP) $(0,5,10$, and 15, $25 \mathrm{~cm}$ of $\mathrm{H} 2 \mathrm{O})$ using Krypton $\left(\mathrm{Kr}^{81 \mathrm{~m}}\right)$.

Results: Two of the four infants showed unilateral areas of absent ventilation at that improved with higher PEEP (figure). The areas involved correlated with the findings on dynamic TBG.
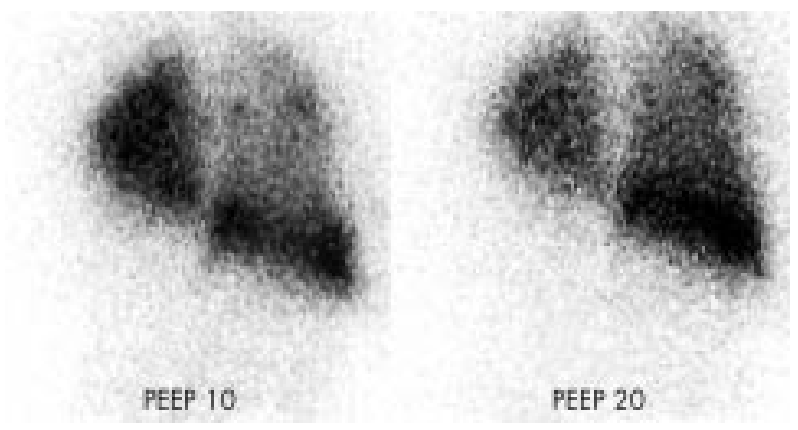

Conclusions: Krypton lung scans with variable PEEP is a sensitive technique to diagnose TBM. It has an extremely low radiation burden and is non invasive. Furthermore it also gives an indication of peripheral or localised forms of bronchomalacia which can be missed on dynamic TBG. The TBG gives a detailed anatomical picture of the airway, while the radio nucleotide ventilation scan provides physiological images of the lungs with the airways held open with known PEEP levels. A prospective study is currently underway to determine sensitivity and specificity of IVS in tracheobronchomalacia. 